\title{
Entanglement, partial set of measurements, and diagonality of the density matrix in the parton model
}

\author{
Vladimir Skokov ${ }^{a, b, *}$ \\ ${ }^{a}$ Department of Physics, North Carolina State University, \\ Raleigh, NC 27695, USA \\ ${ }^{b}$ Riken-BNL Research Center, Brookhaven National Laboratory, \\ Upton, NY 11973, USA \\ E-mail: vskokov@ncsu.edu
}

\begin{abstract}
We consider the following conundrum which attracted attention recently: on one hand the proton as a quantum object is in a pure state and is described by a completely coherent wave function with zero entropy. On the other hand in high energy experiments (DIS) when probed by a small external probe, it behaves like an incoherent ensemble of (quasi-free) partons.

In this talk, we define the "entropy of ignorance," which quantifies the entropy associated with the ability to perform only a partial set of measurements on a quantum system. For a parton model, the entropy of ignorance is equal to a Boltzmann entropy of a classical system of partons. We analyze a calculable model used for describing low x gluons in the Color Glass Condensate approach, which has similarities with the parton model of QCD. In this model we calculate the entropy of ignorance in the particle number basis as well as the entanglement entropy of the observable degrees of freedom. We find that the two are similar at high momenta, but differ by a factor of order unity at low momenta. This holds for the Renyi as well as von Neumann entropies. We conclude that entanglement does not seem to play an important role in the context of the parton model.
\end{abstract}

HardProbes 2020

1-6 June 2020

Austin, Texas

${ }^{*}$ Speaker 


\section{Introduction}

In reference [1], the authors put forward one possible resolution of the following paradox. On one hand the proton as a quantum object in a pure state and as such has zero entropy. On the other hand in high energy experiments (DIS) the measurements are well described by an incoherent ensemble of (quasi-free) partons with a nonvanishing "classical" entropy. The suggested resolution is that the origin of this entropy is entanglement between the observed in DIS degrees of freedom and the rest of the proton wave function which are not measured in the final state and therefore play the role of an "environment". Namely, if one knew the proton wave function, one would be able to calculate this density matrix by reducing it with respect to the "environment": $\hat{\rho}_{\mathrm{PM}}=\operatorname{Tr}_{\text {unobs }}[|P\rangle\langle P|]$. The entropy of the parton model is then identified with the von Neumann entropy of the reduced density matrix according to $S_{\mathrm{PM}}=-\operatorname{Tr}\left[\hat{\rho}_{\mathrm{PM}} \ln \hat{\rho}_{\mathrm{PM}}\right]$. However there is another natural resolution [2]. The set of measurements that is described by the parton model is not complete, in the sense that it does not provide full information about the density matrix. In DIS, it is possible to measure the average number of particles and transverse momentum distributions (including multiparticle ones). The problem is that all of these observables are diagonal in the number operator basis, and therefore carry no information about nondiagonal elements of the density matrix in this basis. That is one may come up with an infinite number of density matrices; all of them will be completely equivalent for the limited purpose of describing the results of only these measurements.

Interestingly, this lack of knowledge of the actual density matrix of the system can be characterized by an entropy. We will dub this entropy "the entropy of ignorance". A particularly amusing case for the entropy of ignorance arises if we consider a system in a pure state. In this case, the von Neumann entropy is strictly zero; however if we ignore the off-diagonal elements of the density matrix and compute the entropy of ignorance the result is non-zero.

We will consider the proton wave function in the Color Glass Condensate (CGC) effective theory. The CGC describes scattering at high energy. For an ultra relativistic hadron, large fraction of momentum is carried by the valence quarks and gluons. Due to their quantum nature, partons carrying large fraction of momentum radiate low energy gluons which have a lifetime relatively short to that of the valence charges. The CGC wave function was introduced and derived in Refs. [3, 4]. The wave function of the system of slowly evolving valence charges and faster soft gluon degrees of freedom has the form $|\psi\rangle=|s\rangle \otimes|v\rangle$, where $|v\rangle$ is the state vector characterizing the valence degrees of freedom and $|s\rangle$ is the vacuum of the soft fields in the presence of the valence source. We use the McLerran-Venugopalan (MV) model for the valence degrees of freedom and the leading order CGC wave function for soft fields, see Ref. [2] for details.

The hadron density matrix $\hat{\rho}=|v\rangle \otimes|s\rangle\langle s| \otimes\langle v|$ describes a pure quantum state. We can integrate out the valence (slow) degrees of freedom and define the reduced density matrix for the soft gluons:

$$
\hat{\rho}_{r}=\operatorname{Tr}_{\rho} \hat{\rho} \equiv \int D \rho\langle\rho|\hat{\rho}| \rho\rangle=\int D \rho\langle\rho \mid v\rangle|s\rangle\langle s|\langle v \mid \rho\rangle .
$$

We will then use this density matrix for calculating the entanglement entropy of the soft gluons and compare it to the entropy of ignorance. 
Due to the special role of the number of parton representation for the parton model, the calculations will be demonstrated in number of gluons basis. The straightforward calculation [2] leads to

$$
\rho_{n m}=\left\langle n_{c}(\underline{q}), m_{c}(-\underline{q})\left|\hat{\rho}_{r}(\underline{q})\right| \alpha_{c}(\underline{q}), \beta_{c}(-\underline{q})\right\rangle=\frac{(1-R)(n+\beta) !}{\sqrt{n ! m ! \alpha ! \beta !}}\left(\frac{R}{2}\right)^{n+\beta} \delta_{n+\beta, m+\alpha},
$$

where $R=\left(1+\frac{q^{2}}{2 g^{2} \mu^{2}}\right)^{-1}$.

\section{Entropy of ignorance and entropy of entanglement}

The von Neumann entropy of ignorance for a single momentum mode $\underline{q}$ is

$$
\begin{aligned}
& S_{I}(\underline{q})=-\sum_{m, n} \rho_{n m} \ln \rho_{n m} \\
& =-\frac{1}{2}\left(N_{c}^{2}-1\right) S_{\perp} \int_{q} \sum_{m, n}\left[(1-R) \frac{(m+n) !}{m ! n !}\left(\frac{R}{2}\right)^{m+n}\right] \ln \left[(1-R) \frac{(m+n) !}{m ! n !}\left(\frac{R}{2}\right)^{m+n}\right] .
\end{aligned}
$$

This expression does not have a closed analytical form [2].

The entanglement entropy in this model was calculated for the first time in Ref. [5]. It also can be reproduced using the formalism in the previous section. The complete final result is

$$
\begin{aligned}
S_{E} & =-\operatorname{Tr} \hat{\rho}_{r} \ln \hat{\rho}_{r}=\frac{1}{2}\left(N_{c}^{2}-1\right) S_{\perp} \\
& \times \int \frac{d^{2} q}{(2 \pi)^{2}}\left[\ln \left(\frac{g^{2} \mu^{2}}{q^{2}}\right)+\sqrt{1+4 \frac{g^{2} \mu^{2}}{q^{2}}} \ln \left(1+\frac{q^{2}}{2 g^{2} \mu^{2}}+\frac{q^{2}}{2 g^{2} \mu^{2}} \sqrt{1+4 \frac{g^{2} \mu^{2}}{q^{2}}}\right)\right] .
\end{aligned}
$$

There are a few notable properties in this expression:

- In general, for an arbitrary momentum the entanglement entropy may not be represented in the conventional form for Bose particles:

$$
S=\left(N_{c}^{2}-1\right) S_{\perp} \int \frac{d^{2} q}{(2 \pi)^{2}}\left[\left(n_{q}+1\right) \ln \left(n_{q}+1\right)-n_{q} \ln n_{q}\right] .
$$

Note that in this equation we included only one gluon polarization, i.e. $2\left(N_{c}^{2}-1\right) \rightarrow\left(N_{c}^{2}-1\right)$, as we are working in the leading density approximation where only longitudinal polarization contributes.

- To gain more insight into Eq. (4), let's analyze it at large momentum. Performing the expansion, it is straightforward to obtain

$$
S_{E}(q) \simeq-\left(N_{c}^{2}-1\right) S_{\perp} \frac{g^{2} \mu^{2}}{q^{2}} \ln \left(\frac{g^{2} \mu^{2}}{q^{2}}\right),
$$

where $S_{E}(q)$ is defined through $S_{E}=\int \frac{d^{2} q}{(2 \pi)^{2}} S_{E}(q)$. Identifying $n_{q}$ with the gluon WilliamsWeizsäcker (WW) occupation number $n_{q}=\left\langle a^{\dagger}(q) a(q)\right\rangle=\frac{g^{2} \mu^{2}}{q^{2}}$, we obtain

$$
S_{E}(q)=-\left(N_{c}^{2}-1\right) S_{\perp} n_{q} \ln n_{q}
$$




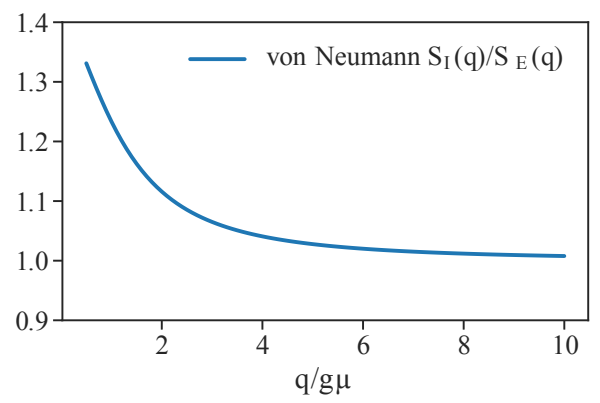

Figure 1: Ratios of the entropy densities at a given magnitude of the transverse momentum $q / g \mu$.

which reproduces Eq. (5) in the limit of the low occupation number. This shows that at high momentum WW gluons are appropriate degrees of freedom characterizing the entanglement entropy density.

- At small momenta, one gets

$$
S_{E}(q) \simeq-\left(N_{c}^{2}-1\right) S_{\perp}\left[\ln \left(\frac{g \mu}{q}+1\right)+\frac{g \mu}{q} \ln \left(1+\frac{q}{g \mu}\right)\right]
$$

or, identifying $n_{q}=\frac{g \mu}{q}$

$$
S_{E}(q)=\left(N_{c}^{2}-1\right) S_{\perp}\left[\ln \left(n_{q}+1\right)+n_{q} \ln \left(1+1 / n_{q}\right)\right] .
$$

This modulo rearrangement of the terms coincides with Eq. (5). It is important to notice that the identification $n_{q}=\frac{g \mu}{q}$ is different from the WW occupation number which is given by $\frac{g^{2} \mu^{2}}{q^{2}}$ at all momenta. To understand this better, consider Bose-Einstein thermal distribution for massless particles in the limit of small momenta $q \ll T: n_{q}=\frac{1}{\exp \left(\frac{q}{T}\right)-1} \approx \frac{T}{q}$. Thus $n_{q}=\frac{g \mu}{q}$ coincides with the thermal distribution at small momenta, if we assign $T$ to $g \mu$. Amusingly this suggests that due to entanglement the systems is thermal at small momenta and that the appropriate degrees of freedom are not WW gluons! This statement can be made more precise and proven rigorously by performing canonical transformations and defining a Bose quasi-particle state, for details see Ref. [6].

\section{Discussions and Conclusions}

Comparing the ignorance and entanglement entropies at large $q$ to the sub-leading

$$
S_{I}(q) \simeq \frac{\left(N_{c}^{2}-1\right) g^{2} \mu^{2} S_{\perp}}{q^{2}}\left[\ln \left(e \frac{q^{2}}{g^{2} \mu^{2}}\right)+\frac{g^{2} \mu^{2}}{q^{2}} \ln \frac{e}{2}\right]
$$

and

$$
S_{E}(q) \simeq \frac{\left(N_{c}^{2}-1\right) g^{2} \mu^{2} S_{\perp}}{q^{2}}\left[\ln \left(e \frac{q^{2}}{g^{2} \mu^{2}}\right)-\frac{g^{2} \mu^{2}}{q^{2}} \ln \left(e \frac{q^{4}}{g^{4} \mu^{4}}\right)\right]
$$


we conclude that the leading behavior of the two expressions is the same. The difference appears in a subleading power of $1 / q^{2} \ln q^{2}$. For an arbitrary momentum, the ratio of two entropies is shown in Fig. 1.

The differences between $S_{I}$ and $S_{E}$ disappear in the ultraviolet. A careful analysis that the identical UV asymptotics of $S_{I}\left(q^{2}\right)$ and $S_{E}\left(q^{2}\right)$ is due to the small occupation numbers of partons at large $q^{2}$. Indeed, at intermediate and low momenta where the occupation numbers per unit phase space volume are of order unity the difference between the two types of entropies becomes significant, at the order of 50\%. We expect that the real parton model of QCD shares these features. At very large momenta the entanglement and ignorance lead to the same entropy, while at low $Q^{2}$ the resulting entropies should be different. This is likely to be unrelated to any nontrivial dynamics of the "environment" degrees of freedom, such as confinement but is just the consequence of low occupation number of partons at high momentum. To summarize, the lack of coherence and large entropy of the partonic density matrix within the parton model approach must be due to "ignorance", i.e. to our ability to measure only a restricted number of observables, rather than to the entanglement of the observed partons with the unobserved degrees of freedom, as suggested in Ref. [1].

\section{References}

[1] D.E. Kharzeev and E.M. Levin, Deep inelastic scattering as a probe of entanglement, Phys. Rev. D95 (2017) 114008 [1702.03489].

[2] H. Duan, C. Akkaya, A. Kovner and V.V. Skokov, Entanglement, partial set of measurements, and diagonality of the density matrix in the parton model, Phys. Rev. D 101 (2020) 036017 [2001.01726].

[3] A. Kovner, M. Lublinsky and U. Wiedemann, From bubbles to foam: Dilute to dense evolution of hadronic wave function at high energy, JHEP 06 (2007) 075 [0705. 1713].

[4] T. Altinoluk, A. Kovner, M. Lublinsky and J. Peressutti, QCD Reggeon Field Theory for every day: Pomeron loops included, JHEP 03 (2009) 109 [0901. 2559].

[5] A. Kovner and M. Lublinsky, Entanglement entropy and entropy production in the Color Glass Condensate framework, Phys. Rev. D92 (2015) 034016 [1506. 05394].

[6] H. Duan, A. Kovner and V.V. Skokov, "to be published.". 